\title{
Total Occlusion of the Internal Carotid Artery by Subacute In-Stent Thrombosis and Subsequent Spontaneous Recanalization After Stent-Assisted Coil Embolization
}

\author{
Cheol Young Lee, MD', Chang-Woo Ryu, MD², Jun Seok Koh, MD', Eui Jong Kim, MD³
}

We present a 61-year-old female with an unruptured intracranial aneurysm on the left superior hypophyseal artery. The patient was treated with endovascular management, stent-assisted coil embolization. Multiple embolic infarction and total occlusion of the left internal carotid artery (ICA) was occurred one day later. At 14 days of heparinization, complete recanalization of the ICA and full recovery of neurologic symptoms were achieved. Stent-assisted coil embolization is not without risk of instent thrombosis and the subsequent embolism. The cause of subacute in-stent thrombosis and natural course were uncertain; however, the clinical course may potentially be fatal. Therefore, rapid diagnosis and proper treatment are recommended.

Key Words : Subacute thrombosis; Recanalization; Stent-assisted coiling

Currently, the stent has been used widely in various diseases such as cervical carotid stenosis, intracranial stenosis, dissecting aneurysms and intracranial wideneck aneurysms, where stents are placed across the neck of an aneurysm to allow for coil deployment into the aneurysm without compromising the parent vessel. Tthromboembolic events are known to be the most

\footnotetext{
'Departments of Neurosurgery and ${ }^{2}$ Radiology, Kyung Hee University Hospital at Gangdong, Seoul, Korea

${ }^{3}$ Department of Radiology, Kyung Hee University Hospital, Seoul, Korea

Received September 13, 2010;

accepted after revision December 24, 2010.

Correspondence to: Jun Seok Koh, MD, Department of Neurosurgery, Kyung Hee University Hospital at Gangdong, 892 Dongnam-ro, Sangil-dong, Gangdong-gu, Seoul 134-090, Korea.

Tel. 82-2-440-6145 Fax. 82-2-440-7171

E-mail: gjs@khnmc.or.kr

Neurointervention $2011 ; 6: 38-41$

This is an Open Access article distributed under the terms of the Creative Commons Attribution Non-Commercial License (http://creativecommons.org/licenses/by-nc/3.0) which permits unrestricted non-commercial use, distribution, and reproduction in any medium, provided the original work is properly cited.
}

frequent complications of stent treatment. However, subacute in-stent thrombosis after stenting procedures is a rare complication, which was reported in only one of 146 procedures in previous reports. Its course is often potentially fatal. However, the natural course and optimal treatment have not been discussed in detail (1). Unfortunately, the physiologic mechanism of these serious procedure-related complications, such as instent stenosis or subacute in stent thrombosis after stenting and coiling of aneurysms, and their influence on overall long-term outcomes is unknown. Some authors have reported on cases treated by thrombolysis or in-stent percutaneous transluminal angioplasty (PTA) and others experienced spontaneous recanalization (2). Spontaneous recanalization of occluded internal carotid artery (ICA) does not frequently occur. The natural course of a proximal ICA occlusion and its possibility for recanalization, including the exact time of recanalization after occlusion, are not well known $(3,4)$. A standard strategy for management of these complications has not been established. Outcomes after successful recanalization remain unclear. Here, we 
described a case of stent-assisted coiling complicated with subacute in-stent thrombosis by spontaneous recanalization without thrombolysis or PTA, and attempt to discuss how often subacute in-stent thrombosis occurs after treatment, under what circumstances this complication can be expected, and how it can be effectively treated.

\section{CASE REPORT}

A 61-year-old female patient, who had coronary artery disease with a recent headache, tinnitus and dizziness visited our medical center. On examination by magnetic resonance imaging (MRI), a large aneurysm was found incidentally in the supraclinoid area and the patient was referred to our department for evaluation. Three-dimensional (3D) computed tomographic angiog- raphy revealed a large unruptured aneurysm measuring $14 \mathrm{~mm}$ in the left distal ICA. On conventional angiography, the aneurysm was confirmed as a left superior hypophyseal artery aneurysm $(12 \times 18 \mathrm{~mm}, 6.5 \mathrm{~mm}$ neck) (Fig. 1A). Two 2 weeks later, the patient was admitted for endovascular coiling of an unruptured aneurysm. Due to heart disease, she had already taken antiplatelet agents. Under general anesthesia, neurophysiological monitoring was used for all procedures. After access to the femoral artery was obtained with a $7 \mathrm{~F}$ angiosheath, a bolus of 4,000 IU heparin was administered in order to increase the activated clotting time to twice that of the baseline $(250 \sim 300 \mathrm{sec})$. Left carotid catheterization was performed with a 7F guiding catheter (Guider Softip ${ }^{\mathrm{TM}}$ XF, Boston Scientific, Plymouth, Massachusetts, USA), microcatheter (Excelsior SL-10, pre-shaped; Boston

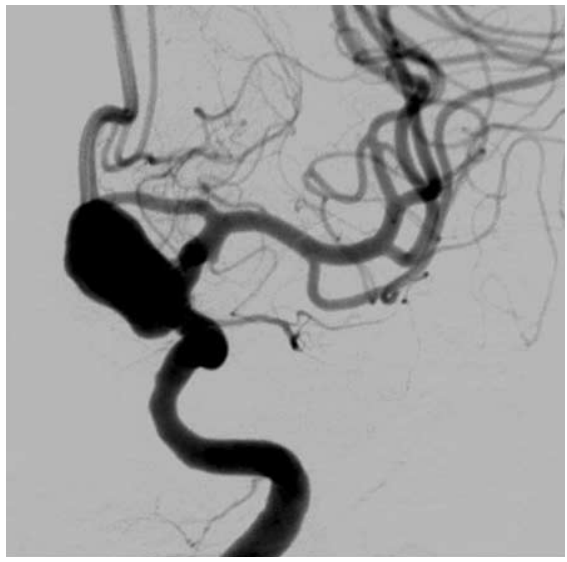

A

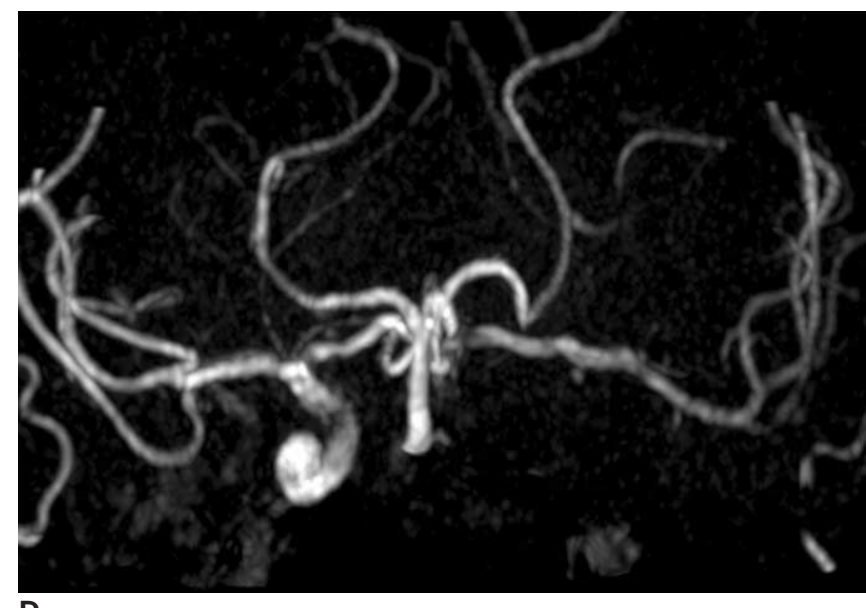

D
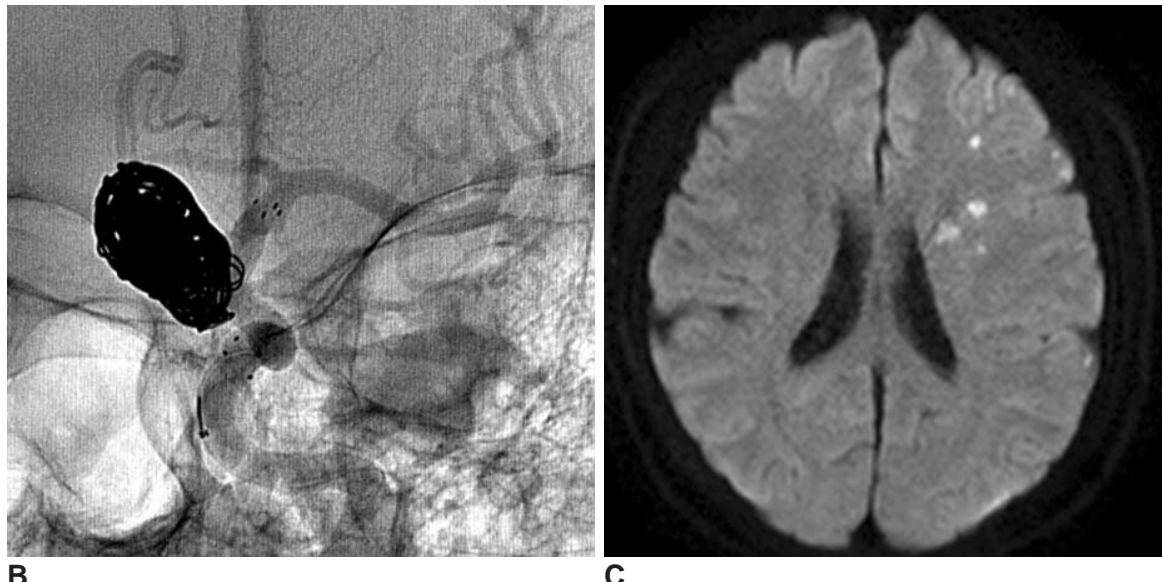

C

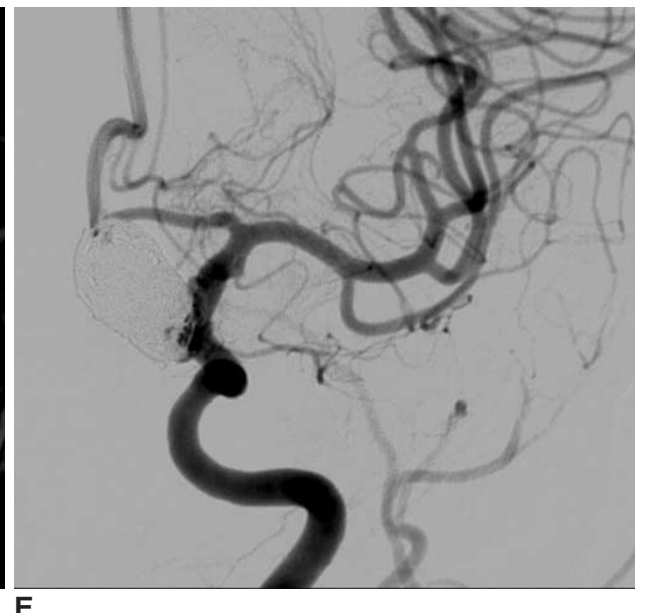

Fig. 1. A. A 61-year-old female, 3-dimensional rotation angiography demonstrates a left superior hypophyseal aneurysm.

B. After insertion of 7 coils, two small loops protruded into the parent artery lumen. However, 3D-rotational angiography (not seen) demonstrated that coil loops were located between the parent artery and the stent.

C. One day after the procedure, diffusion-weighted MR revealed focal multiple acute infarction due to a distal thromboembolism.

D. MR angiography showed total occlusion of the distal ICA. However, cross filling was observed throughout the anterior communicating artery.

E. At 2 weeks postoperatively, complete recanalization was confirmed in conventional angiography. 


\section{Cheol-Young Lee, et al.}

Scientific, Fremont, California, USA) and microwire were passed to the distal ICA, and the tip of microcatheter was put into the aneurysm sac. The first detachable coil was inserted into the aneurysm sac; however, protrusion into the parent artery was happened occurred despite several attempts at repositioning. The jailing technique with stent was planned. The microcatheter and microwire complex was navigated in to the distal ICA and passed through the aneurismal neck for stenting. A self-expander stent (Neuroform stent, $4.5 \times 30 \mathrm{~mm}$; Fremont, California, USA) was deployed across the neck. After confirming ICA patency and the shape of stent, the first coil was inserted in order to make a basket. An additional 9 coils were inserted into the aneurysm sac (Fig. 1B). $3 \mathrm{D}$-rotational angiography confirmed that the coil loops were located between the parent artery and the stent. Lt. ICA angiogram showed nearly complete embolization of the aneurysmal sac and good patency of the ICA. The patient's neurologic status did not change after the procedure. One day after the procedure, the patient experienced symptoms of transient ischemic attack, right mild hemiparesis and dysarthria for a few minutes. Diffusion-weighted MRI and MR angiogram (MRA) revealed a focal multiple acute infarctions due to a distal thromboembolism and total occlusion of the distal ICA. However, cross filling was observed through the anterior communicating artery (Fig. 1C, D). Due to the mildness of neurologic symptoms and good collateral filling, systemic heparinization was started rather than direct thrombolysis. Neurologic symptoms showed improvement over time, and the patient showed complete recoveryat 4 days postoperatively, follow up perfusion MRI and MRA showed partial recanalization of totally occluded ICA and no perfusion defect in the left cerebral hemisphere. At 2 weeks postoperatively, complete recanalization was confirmed on conventional angiography, and the patient showed no neurologic deficit (Fig. 1E). Systemic heparinization was stopped at this time. Ten months later, the good patency of the left ICA was shown in MRA.

\section{DISCUSSION}

In the interventional cardiology literature, a number of reports have presented numbers on the occurrence rates of subacute thrombosis in coronary artery stents (3). However, few reports on the incidence, pathophysiology and treatment of subacute in-stent thrombosis have been published in the previous neurointerven- tional literature. Subacute thrombosis is defined by cardiologists as occurring within the first 28 days after the procedure. For bare metal stents, the incidence was in the range of $0.5 \%$ to $1.5 \%$. In drug-eluting stents for coronary artery intervention, subacute thrombosis was reported to be higher compared with bare metal stents $(0.9 \%$ to $3.1 \%)$. Due to this incidence, use of drugeluting stents in intracranial circulation was considered as a current ongoing discussion. Riedel et al. (4) reported that in intracranial stenting, the incidence of subacute in-stent thrombosis was about $10 \%$ of patients, even if they were treated with standardized antiplatelet therapy and suggested that the rate of in stent thrombosis for intracranial circulation is about 7 times higher than that for coronary artery stenting with bare metal stents. This difference between intracranial and coronary subacute in-stent thrombosis might be due to the fact that the intracranial vessel luminal diameter inserted stent is smaller or that the perfusion pressure in coronary artery stenting is higher than that in intracranial vessels. Another reason might be that in coronary stenting, subacute in-stent thrombosis does not always show the dramatic clinical symptoms observed after stent thrombosis in cerebral vessels. Therefore, some subacute coronary stent thrombosis may be unrecognized.

Until now, exact pathophysiology of stent thrombosis has not been identified. A few hypotheses on the reasons for in-stent thrombosis have been proposed. One reason frequently debated by many authors is the low response or non-response to platelet inhibitors (e.g., aspirin or clopidogrel), which has been considered as a major risk factor for developmenting of stent thrombosis during the subacute period after stenting. Reduction or elimination of heparin function after the intervention may result in development stent thrombosis in non-responders during the subacute period. Therefore, many authors have suggested that platelet function in patients who developed subacute thrombotic complications should be analyzed (3-5). Another possible risk factor is the occurrence of atherosclerotic stenosis plaque rupture with release of intrinsic factor promoting thrombosis in patients who underwent treatment for atherosclerotic stenosis. And in those who received stent-assisted coiling, differenrence in stent strut design and potential prolapse of coil segments into the parent artery may be a major risk factor for in-stent thrombosis (4). In addition, inappropriate pretreatment, dissection at the uncovered stent portion, and a high degree of residual stenosis might be important factor in subacute stent thrombosis (5). 


\section{Internal Carotid Artery Occlusion and Spontaneous Recanalization}

Optimal treatments for subacute in-stent thrombosis remain uncertain. Iancu et al. (6) performed immediate intra-arterial thrombolysis instead of rescue surgery in two cases. They used streptokinase in one case, and tenecteplase in the other case. They suggested that a successful recanalization of carotid stent thrombosis did not confer a bad, long-term prognosis, they also suggested rapid invasive diagnosis and reperfusion in case of cerebral ischemia. In our case, Although MRA showed that there was no blood flow in the left ICA by subacute in-stent thrombosis, the patient symptoms were minimal and good crossing filling was observed. Moreover, the patient showed improvement over time. We believed that spontaneous recanalization and collateral circulation occurred after thrombosis. Therefore, anticoagulation therapy was chosen rather than direct thrombolysis. Intravenous heparinization continued for 14 days and complete recanalization and full recovery of neurologic symptoms were achieved.

In cases of ICA dissections, spontaneous recanalization of the ICA has been described as a common phenomenon in about $57 \%$ to $68 \%$ of cases (7). However, it has rarely been reported in patients with an underlying atherosclerotic disease or an embolic cause. The exact timing of spontaneous recanalization remains unclear. However, spontaneous recanalization of occluded ICAs has been reported to occur mainly between one hour to 24 days after the baseline examinations (8). The exact mechanism of spontaneous recanalization, whether it occurs early or late, is not has identified. Several mechanisms, including vasospasm, distal embolization of an occlusive clot, and spontaneous intravascularlysis and fragmentation of the thrombus have been proposed to explain recanalization $(9,10)$. Some authors suggested that the possibility of autolysis in spontaneous recanalization. Spontaneous recanalization is dependent on the composition of the thrombus, and the role of medications and collateral flow in the process of recanalization are unclear (10). Kim et al. (2) reported on one case of spontaneous recanalized ICA. The patient showed were prominent transopthalmic collateral flow, good collateral flow from the contralateral anterior cerebral artery and retrograde flow from the supraclinoid ICA to the occluded proximal ICA. They suggested that retrograde and collateral flow may have had an important role in recanalization.
Finding from this case indicate that stent-assisted coil embolization is not without a risk of in-stent thrombosis and subsequent embolism. The cause of subacute in-stent thrombosis is uncertain. In our case, small coil loop protrusion or possible resistance to antiplatelet drugs maybe related to this complication. In previous reports, the natural course was uncertain, but the clinical course but may be potentially fatal. Therefore, rapid diagnosis and proper treatment should be performed. Treatment modalities include PTA or intravenous thrombolysis, anticoagulants and conservative management. Of these methods, optimal treatment should be considered based on clinical symptoms and radiologic findings.

\section{References}

1. Roubin GS, New G, Iyer SS, Vitek JJ, Al-Mubarak N, Liu MW, et al. Immediate and late clinical outcomes of carotid artery stenting in patients with symptomatic and asymptomatic carotid artery stenosis: a 5-year prospective analysis. Circulation 2001;103:532537

2. Kim EJ, Koh JS, Choi WS. Carotid artery stenting in a patient with spontaneous recanalization of a proximal internal carotid artery occlusion: a case report. Korean J Radiol 2006;7:292-296

3. Smit JJ, van 't Hof AW, de Boer MJ, Hoorntje JC, Dambrink JH, Gosselink AT, et al. Incidence and predictors of subacute thrombosis in patients undergoing primary angioplasty for an acute myocardial infarction. Thromb Haemost 2006;96:190-195

4. Riedel CH, Tietke M, Alfke K, Stingele R, Jansen O. Subacute stent thrombosis in intracranial stenting. Stroke 2009;40:13101314

5. Lesiak M, Grajek S, Pyda M, Skorupski W, Mitkowski P, Grygier $\mathrm{M}$, et al. Subacute stent thrombosis following coronary angioplasty: incidence and risk factors. Kardiol Pol 2003;59:408416

6. Iancu A, Grosz C, Lazar A. Acute carotid stent thrombosis: review of the literature and long-term follow-up. Cardiovasc Revasc Med 2010;11:110-113

7. Sturzenegger M, Mattle HP, Rivoir A, Baumgartner RW. Ultrasound findings in carotid artery dissection: analysis of 43 patients. Neurology 1995;45:691-698

8. Pryor JC, Setton A, Nelson PK, Berenstein A. Complications of diagnostic cerebral angiography and tips on avoidance. Neuroimaging Clin N Am 1996;6:751-758

9. Meves SH, Muhs A, Federlein J, Buttner T, Przuntek H, Postert T. Recanalization of acute symptomatic occlusions of the internal carotid artery. J Neurol 2002;249:188-192

10. Nguyen-Huynh MN, Lev MH, Rordorf G. Spontaneous recanalization of internal carotid artery occlusion. Stroke 2003;34:10321034 